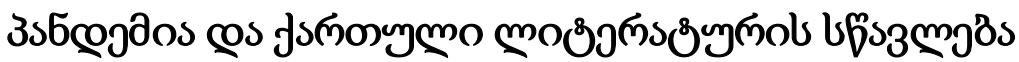

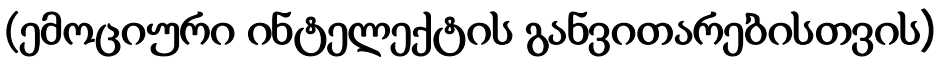

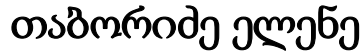

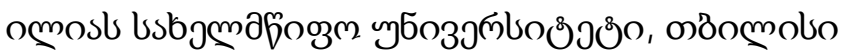

https://doi.org/10.52340/idw.2021.540

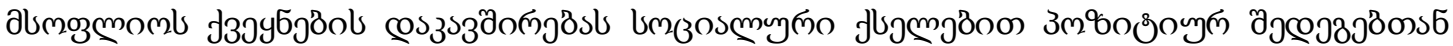

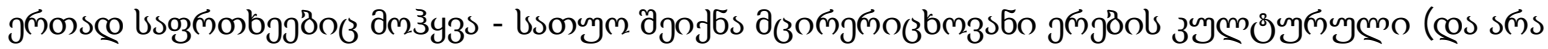

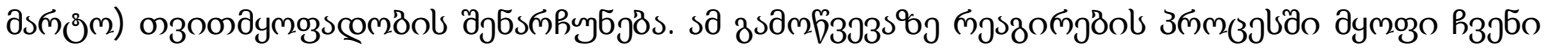

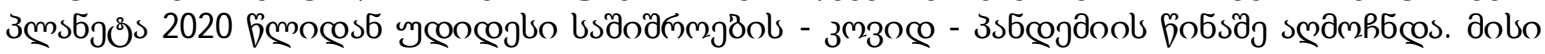

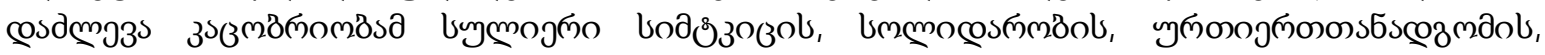

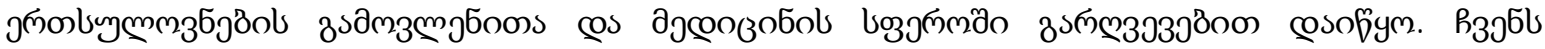

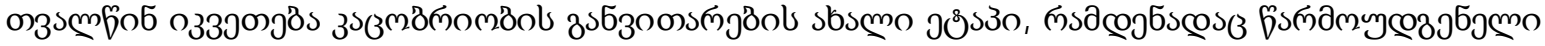

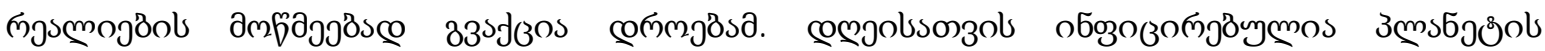

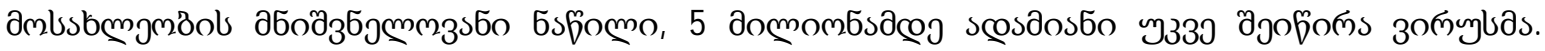

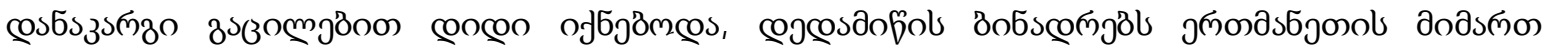

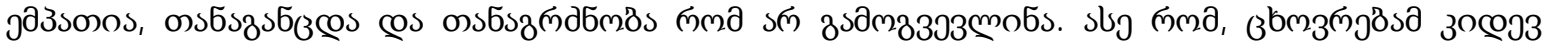

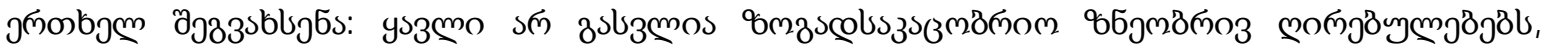

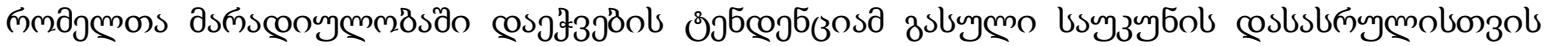

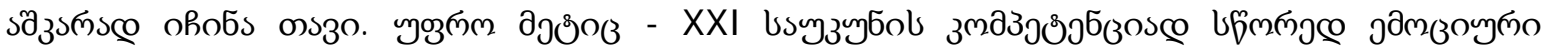

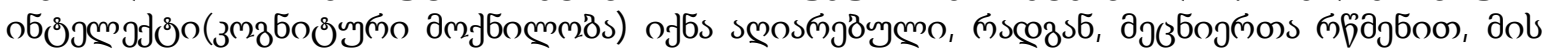

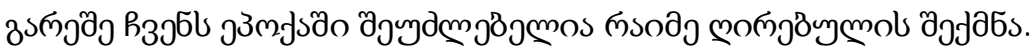

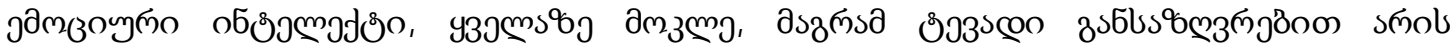

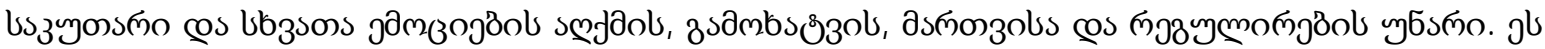

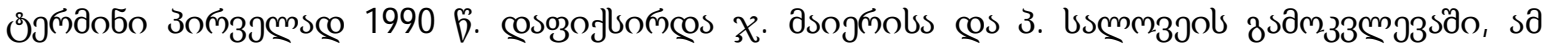

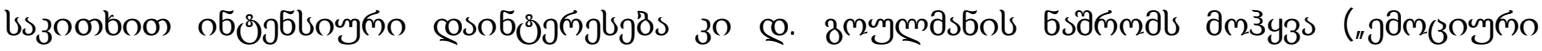

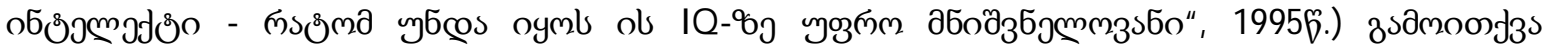

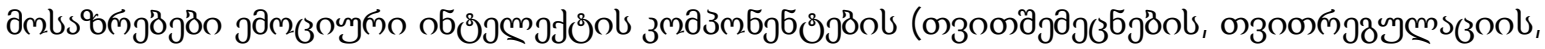

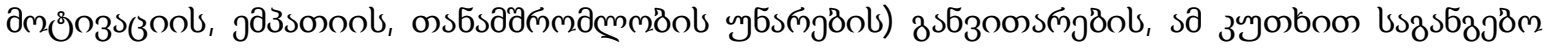

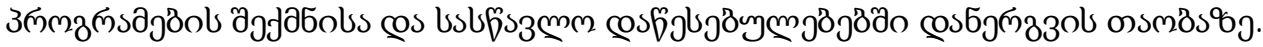

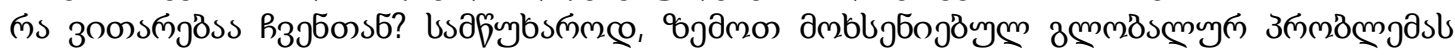

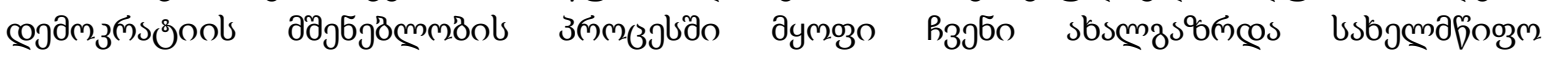

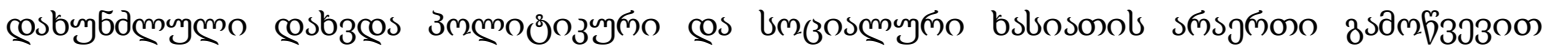

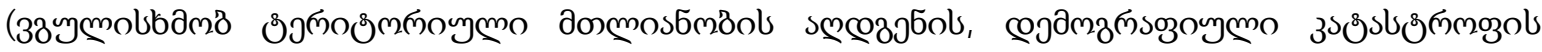

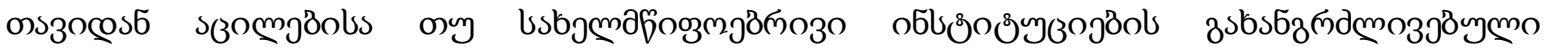

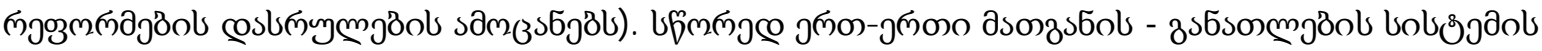

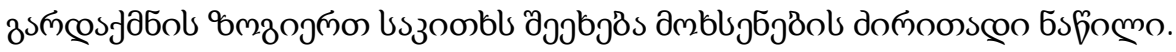

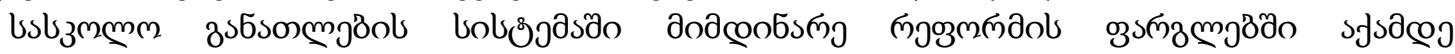

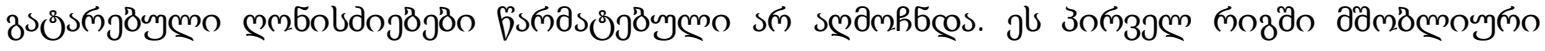

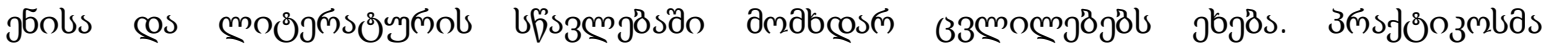

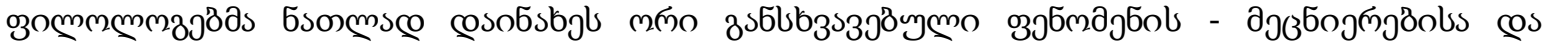

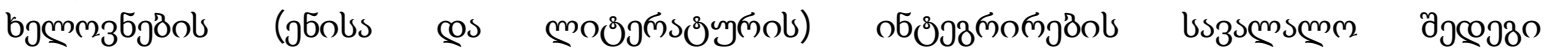

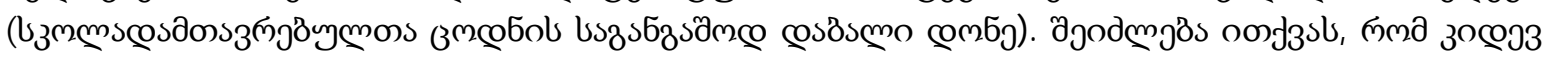




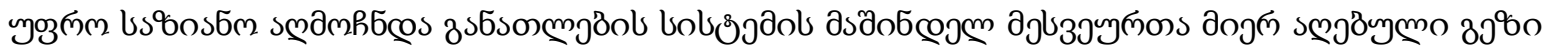

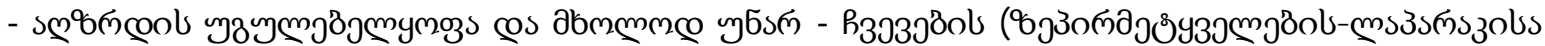

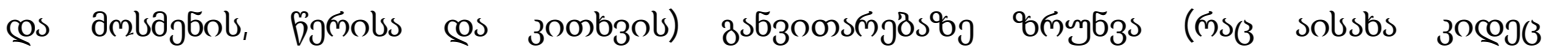

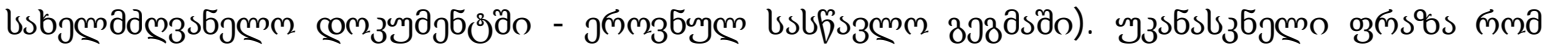

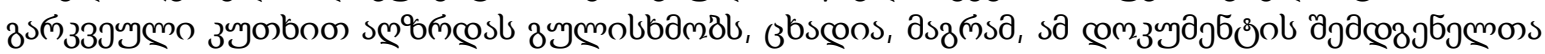

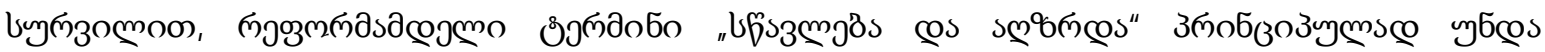

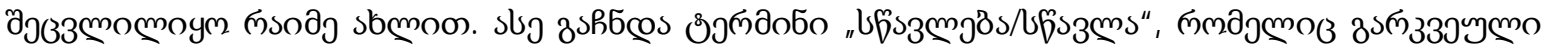

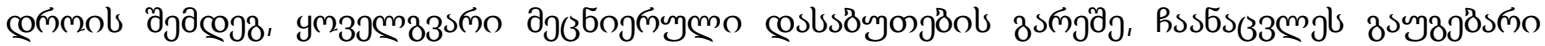

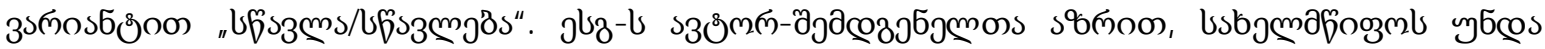

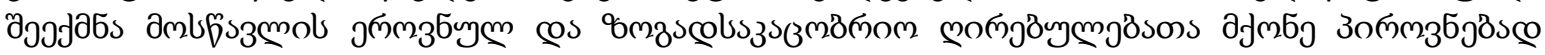

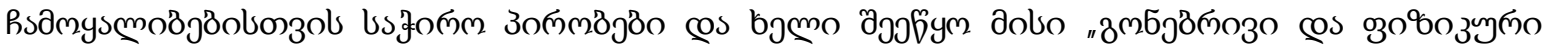

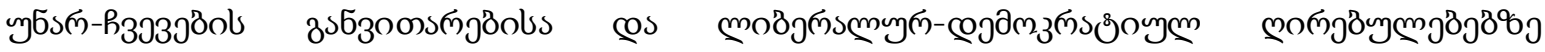

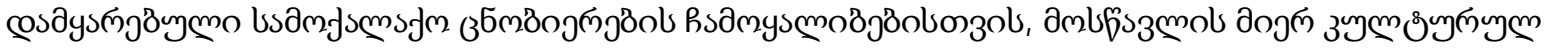

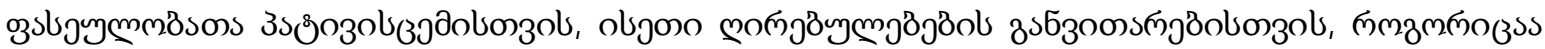

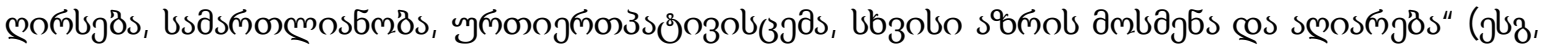

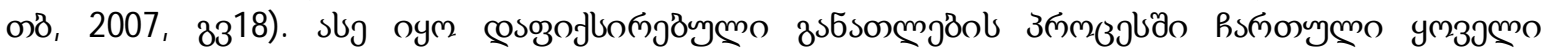

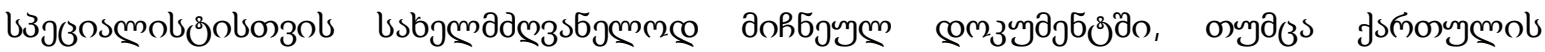

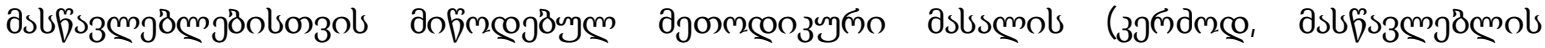

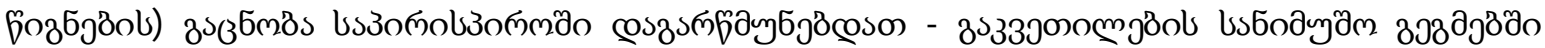

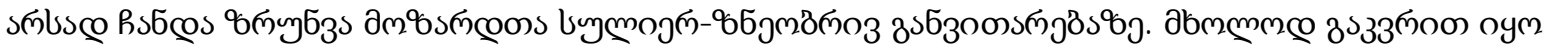

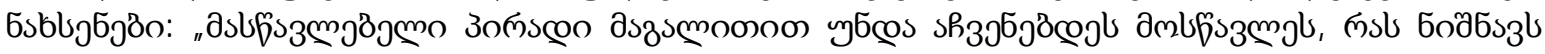

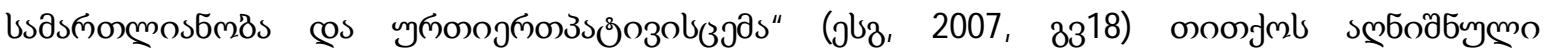

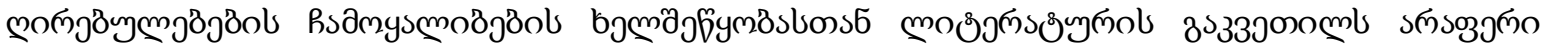

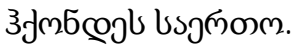

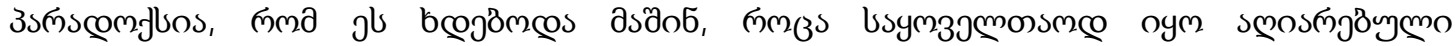

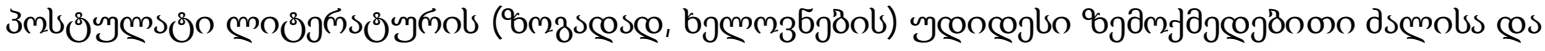

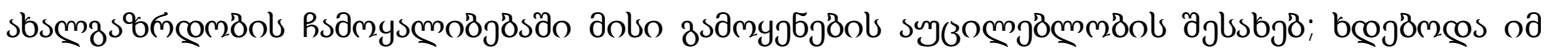

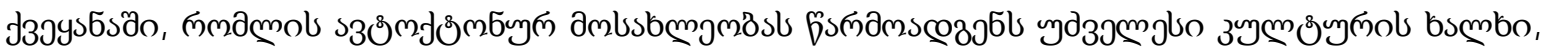

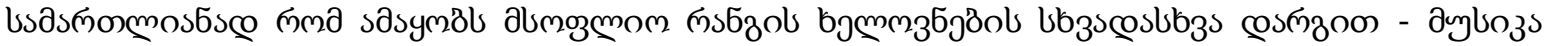

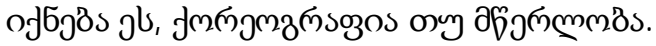

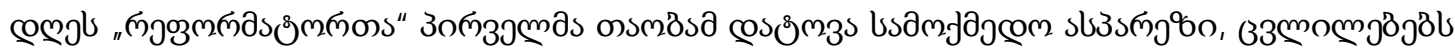

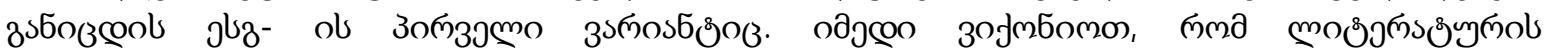

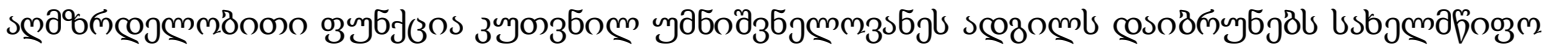

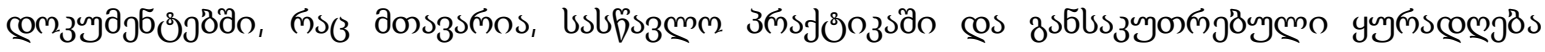

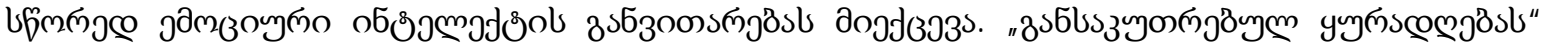

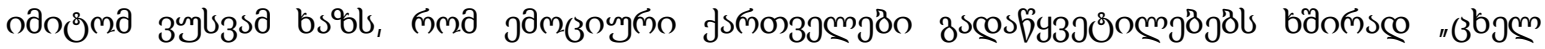

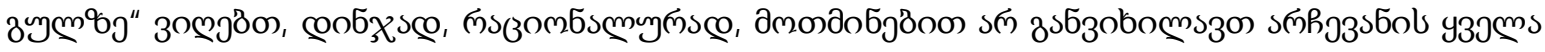

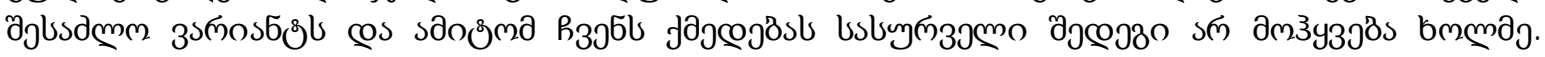

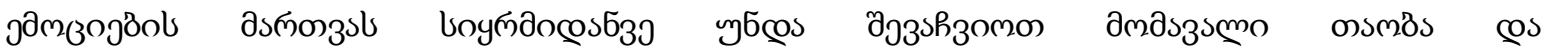

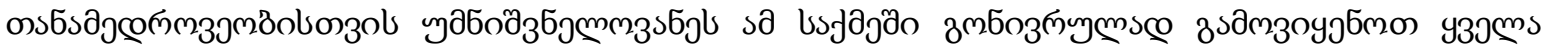

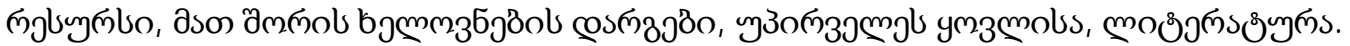

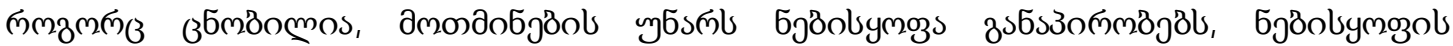

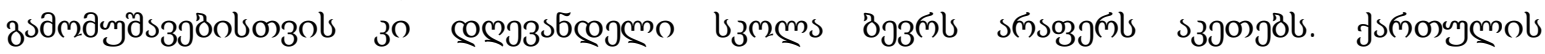

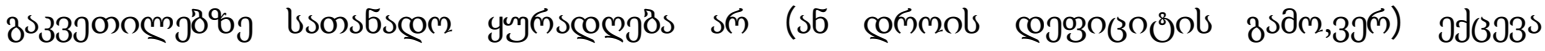

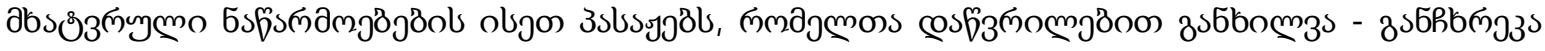

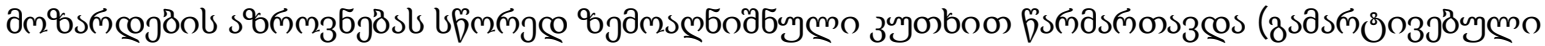

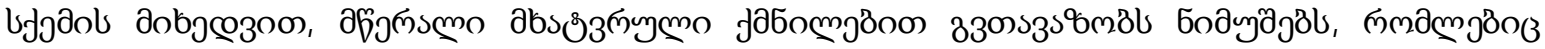

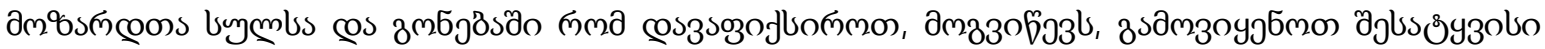

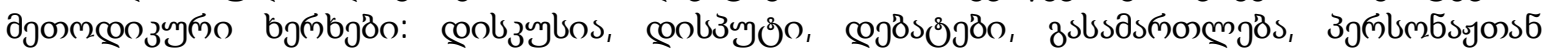

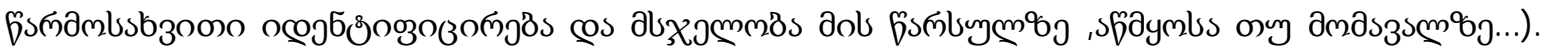




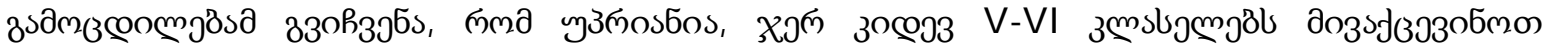

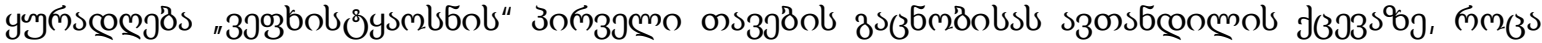

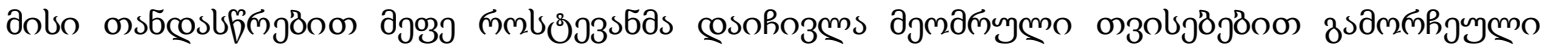

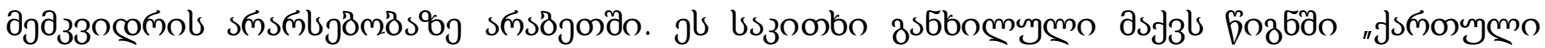

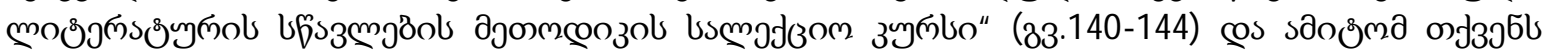

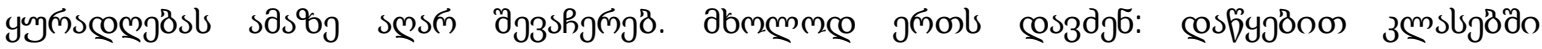

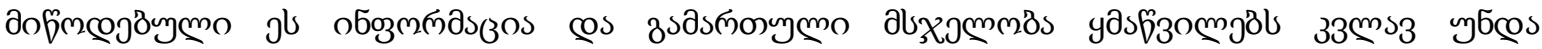

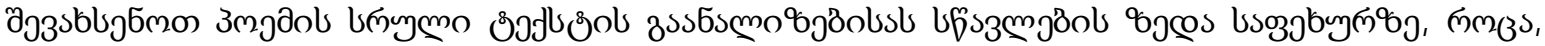

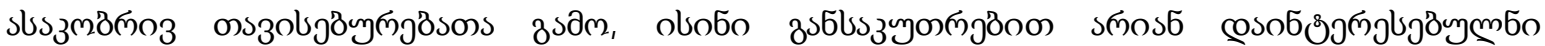

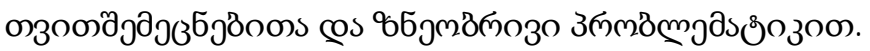

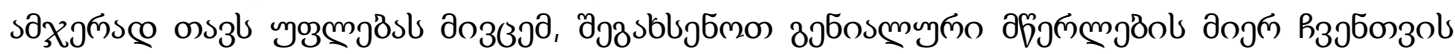

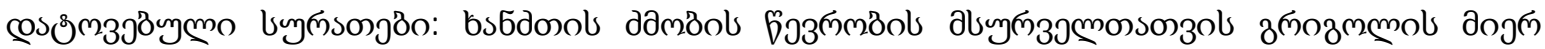

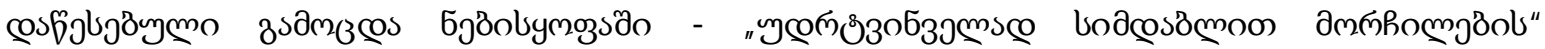

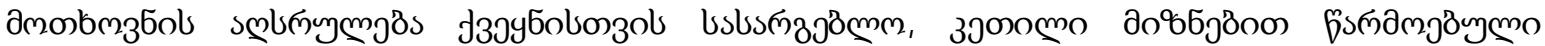

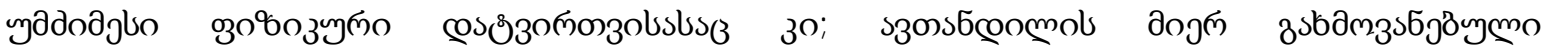

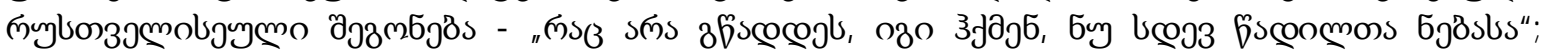

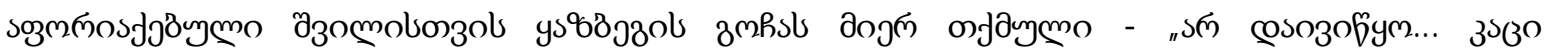

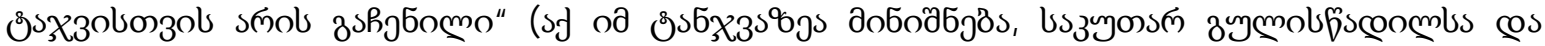

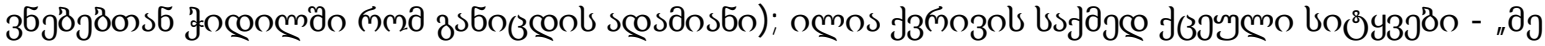

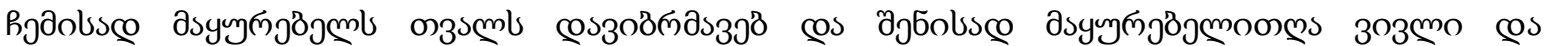

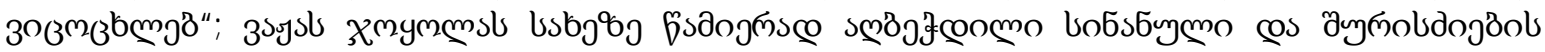

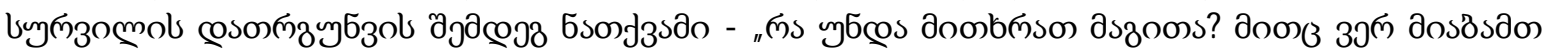

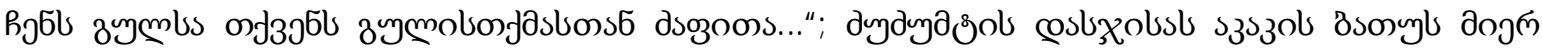

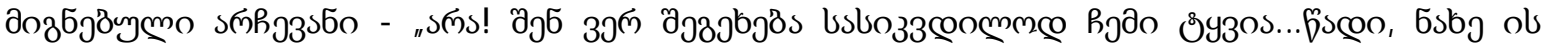

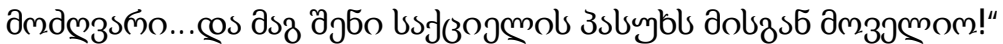

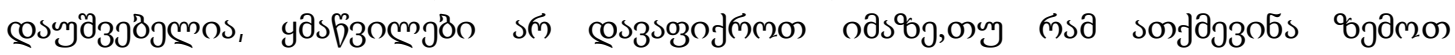

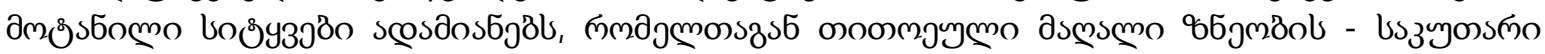

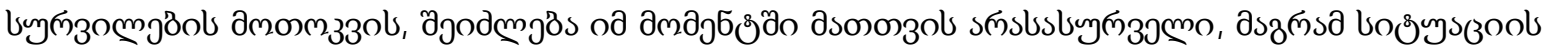

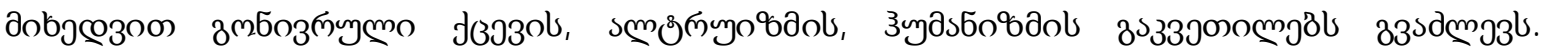

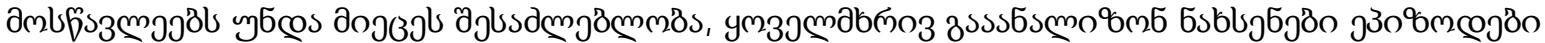

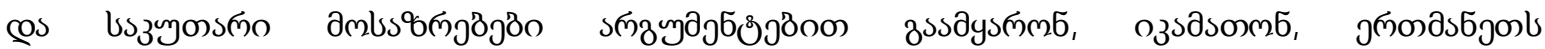

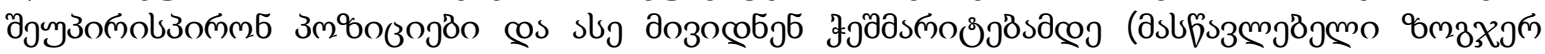

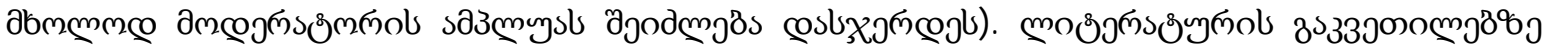

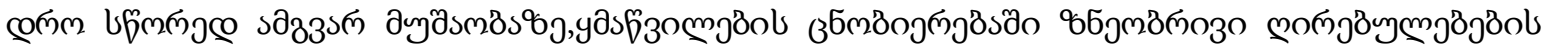

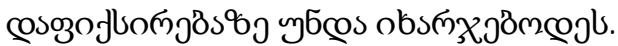

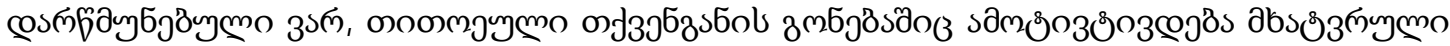

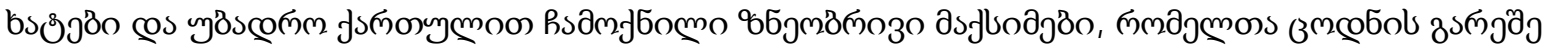

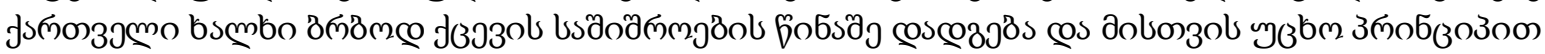

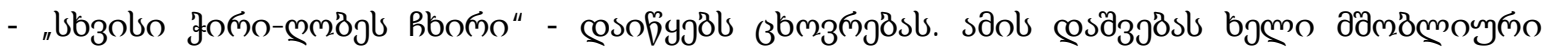

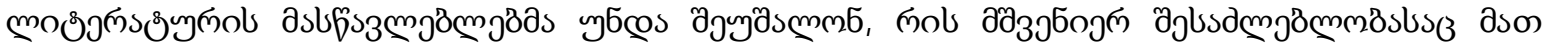

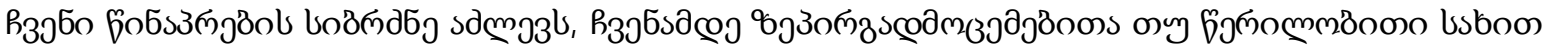

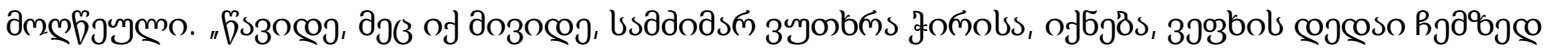

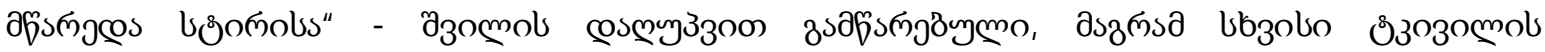
дз

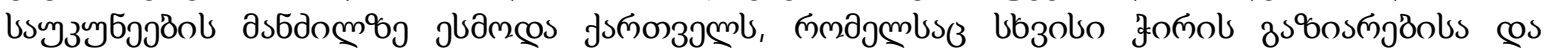

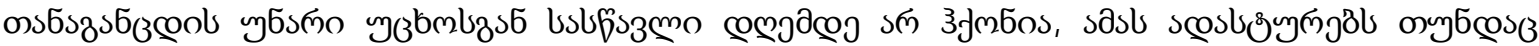

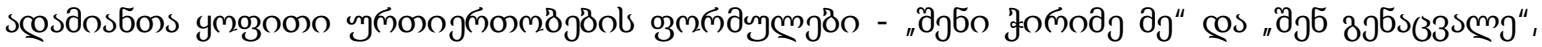

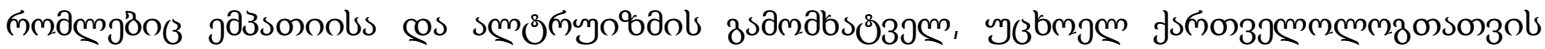

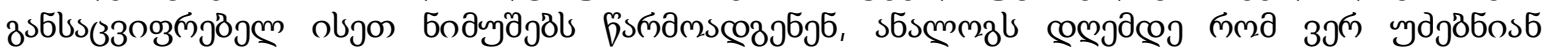

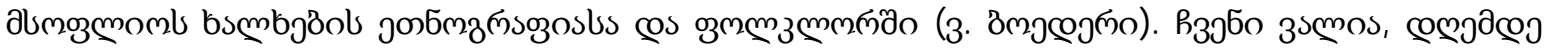




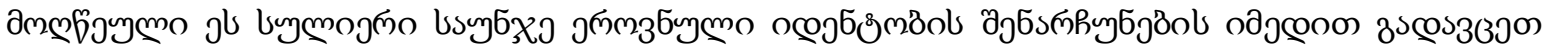

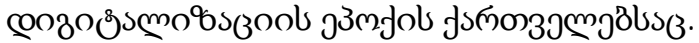

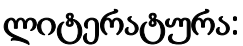

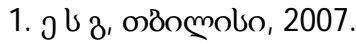

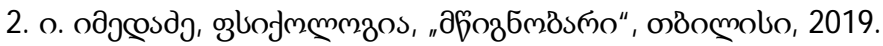

3. Daniel Goleman, Emotional intelligence - W hy it can matter more than IQ, N ew W ork, Bantam books, 1995.

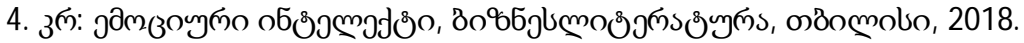

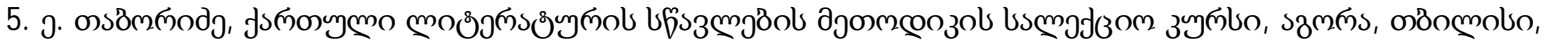
2009.

\title{
Pandemic and Teaching the Georgian Literature (Developing emotional intelligence)
}

Taboridze Elene

Ilia State University,Tbilisi

\begin{abstract}
Since 2020 our planet is facing a huge danger named pandemic Covid-19. This latter seems like is tempting the humankind, reminding that besides technological miracles exist other significant factors necessary for prolonging and supporting the life on earth. Human moral values, like empathy, solidarity, helping those in needed, that were temporarily shifted back to the foreground now naturally moved back to surface. And these highly moral people gifted with such features (mainly medics and volunteers) are fighting against the virus and saving the lives all around the world. Simultaneously, importance and necessity of controlling and managing human emotions in adequate directions have aroused. Thus, emotional intelligence is attracting significant attention, it is the main and necessary background for creating everything that is valuable for humanity.

Either our native country is facing and is fighting against pandemic, the country which is situated on the cross of East-West worlds, the country with enriched and ancient history and culture. After gaining the state independence important institutional branch like educational system was under refining and reforming processes when pandemic as a challenge came upon. Because of latter situation it is of great importance that new vectors and tendencies featured in contemporary worldwide educational systems should be promptly reflected on our educational system as well.

By the author's opinion, main attention should be focused on establishment of highly moral values and emotion controlling issues, especially to an education process of young people born and rising in digitalized epoch. The art, and principally literature, should make significant contribution towards this direction.

In this article some processes and changes made in educational system at schools are discussed and criticized from above stated position; are mentioned and emphasized the centuriesold traditions of preaching humanism, compassion and tolerance in Georgian literature; our literature's possibilities of teaching in terms of the development of emotional intelligence in adolescents are analyze.
\end{abstract}

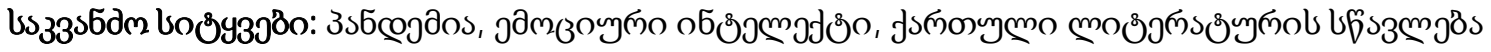

Key Words: Pandemic, emotional intelligence, teaching the Georgian literature 